\title{
THE BRACKET RING OF A COMBINATORIAL GEOMETRY. I
}

\author{
BY
}

NEIL L. WHITE $\left(^{1}\right)$

\begin{abstract}
The bracket ring is a ring of generalized determinants, called brackets, constructed on an arbitrary combinatorial geometry $G$. The brackets satisfy several familiar properties of determinants, including the syzygies, which are equivalent to Laplace's expansion by minors.

We prove that the bracket ring is a universal coordinatization object for $G$ in two senses. First, coordinatizations of $G$ correspond to homomorphisms of the ring into fields, thus reducing the study of coordinatizations of $G$ to the determination of the prime ideal structure of the bracket ring. Second, $G$ has a coordinatization-like representation over its own bracket ring, which allows an interesting generalization of some familiar results of linear algebra, including Cramer's rule.
\end{abstract}

An alternative form of the syzygies is then derived and applied to the problem of finding a standard form for any element of the bracket ring. Finally, we prove that several important relations between geometries, namely orthogonality, subgeometry, and contraction, are directly reflected in the structure of the bracket ring.

1. Introduction. Since combinatorial geometries are most appealing to the intuition when viewed as a generalization of point-sets in finite-dimensional vector spaces, a great deal of effort has been expended toward the generalization of results and concepts of linear algebra to combinatorial geometry. Examples of this might include Higg's work with strong maps, and, more recently, the investigations of Greene, Whiteley, and others into sophisticated exchange properties. However, the lack of actual algebraic structure in combinatorial geometries is the major difficulty.in this process, since all reliance on the scalar field must be first eliminated from any theorem to be generalized.

The bracket ring is an attempt to improve this situation. The brackets, which behave like determinants, are used in place of scalars, for example, as coefficients of linear dependence in Theorem 5.2. This approach agrees in spirit very closely with that of Whiteley [15], who proves that all theorems in the

Presented to the Society, January 26, 1973; received by the editors August 7, 1973.

AMS (MOS) subject classifications (1970). Primary 05B25, 05B35, 13A15; Secondary 14M15, 15A15, $15 \mathrm{A75}$.

Key words and phrases. Combinatorial pregeometry, matroid, bracket ring, syzygy, determinant, coordiratization, prime ideal, van der Waerden relation, standard product, transversal geometry, orthogonal geometry, minor.

(1) This research was supported in part by grant NSF-GP-33591X. 
category of coordinatizable combinatorial geometries may be stated and proved in terms of brackets.

A second motivation for the study of the bracket ring also involves an infusion of algebra into combinatorial geometry. We prove that coordinatizations of a geometry correspond to prime ideals in the bracket ring, hence bringing the powerful machinery of commutative ring theory to bear on coordinatization problems.

The ordinary syzygies are borrowed from classical invariant theory. Rota [8] was the first to realize their connection with combinatorial geometries, by noticing that they embodied the basis exchange axiom.

Our present work is primarily concerned with building the background theory of the bracket ring-its coordinatization properties, its use in place of a scalar field to represent the geometry, and various aspects of its structure. This theory is then applied in the sequel [14] to the particular case of unimodular geometries.

2. Combinatorial pregeometries. We present here some preliminary definitions and propositions, with proofs omitted. Further details may be found in [1].

A combinatorial pregeometry (or matroid) $G(S)$ is a set $S$ together with a nonempty collection $B$ of finite subsets of $S$, called bases, satisfying

(i) $A, B \in B, A \neq B \Rightarrow A \not \subset B$.

(ii) (Exchange Axiom). $A, B \in B, b \in B \Rightarrow$ there exists $a \in A$ such that $(B-\{b\}) \cup\{a\} \in B$.

Equivalently, (ii) may be replaced by the apparently stronger

(ii') (Simultaneous Exchange Axiom). $A, B \in B, b \in B \Rightarrow$ there exists $a \in A$ such that $(B-\{b\}) \cup\{a\} \in B$ and $(A-\{a\}) \cup\{b\} \in B$. It follows that any two bases of $G(S)$ have the same cardinality, the rank of $G$.

A subset $A$ of $S$ is independent if it is contained in some basis, and dependent otherwise. The rank of a subset $A$ of $S$, or $r(A)$, is the common cardinality of its bases, or maximal independent subsets. A circuit is a minimal dependent subset of $S$. A subset $A$ of $S$ is a flat of $G$ if $A$ has no superset of the same rank. The closure of a subset $A$, denoted $\mathrm{Cl}(A)$, is the unique minimal flat containing $A$.

A pregeometry $G(S)$ is a combinatorial geometry if every subset of $S$ of cardinality at most 2 is independent. For our purposes, the distinction is essentially irrelevant, hence we use the more general term pregeometry throughout this paper.

3. Construction of the bracket ring. Let $G(S)$ be an arbitrary combinatorial pregeometry of rank $n$. To every ordered $n$-tuple $X=\left(x_{1}, x_{2}, x_{3}, \cdots, x_{n}\right)$ of elements of $S$, we associate a symbol $\left[x_{1}, \cdots, x_{n}\right]$, or simply $[X]$, called a bracket. 
In writing any sequence $\left(x_{j}, \cdots, x_{k}\right)$ of elements where only the first and last elements are displayed, the intervening elements are always indexed by unit increments. We also abbreviate $\left(x_{j}, \cdots, x_{k-1}, y, x_{k+1}, \cdots, x_{m}\right)$ by $\left(x_{j}\right.$, $\left.\cdots, x_{m} / x_{k} \rightarrow y\right)$, with multiple replacements denoted similarly. Thus $\left[x_{1}, \cdots\right.$, $\left.x_{n} / x_{i_{1}} \rightarrow y_{1}, \cdots, x_{i_{k}} \rightarrow y_{k}\right]$ denotes the bracket $\left[x_{1}, \cdots, x_{n}\right]$ with $x_{i_{1}}, x_{i_{2}}, x_{i_{3}}, \cdots, x_{i_{k}}$ replaced by $y_{1}, y_{2}, y_{3}, \cdots, y_{k}$, respectively, with the elements being replaced always indexed by sub-subscripts of unit increment. If the elements in $X=\left(x_{j}, \cdots, x_{k}\right)$ are distinct, we let $X$ denote the set $\left\{x_{1}\right.$, $\left.\cdots, x_{k}\right\}$ as well as the sequence, where no confusion will arise. If $X=$ $\left(x_{1}, \cdots, x_{n}\right)$ and $\sigma$ is a permutation of $\{1, \cdots, n\}$, we denote the bracket $\left[x_{\sigma 1}, \cdots, x_{\sigma n}\right]$ by $[\sigma X]$.

Let $R_{G}$ be the polynomial ring over the integers generated by the indeterminates $\left\{[X]: X \in S^{n}\right\}$. Let $I_{G}$ be the ideal of $R_{G}$ generated by all elements of the following four types:

(1) $[X]$, if $X$ contains repeated elements,

(2) $[X]$, if the elements of $X$ are distinct but are dependent in $G$,

(3) $[X]-(\operatorname{sgn} \sigma)[\sigma X]$ for any permutation $\sigma$ of $\{1, \cdots, n\}$,

(4) $\left[x_{1}, \cdots, x_{n}\right]\left[y_{1}, \cdots, y_{n}\right]-\Sigma\left[y_{i_{1}}, \cdots, y_{i_{k}}, x_{k+1}, \cdots, x_{n}\right]$. $\left[y_{1}, \cdots, y_{n} / y_{i_{1}} \rightarrow x_{1}, \cdots, y_{i_{k}} \rightarrow x_{k}\right]$, for any $k \leqslant n$ and $x_{1}, \cdots, x_{n}$, $y_{1}, \cdots, y_{n} \in S$, where the sum is over all $i_{1}, \cdots, i_{k}$ such that $1 \leqslant i_{1}<i_{2}$ $<\cdots<i_{k} \leqslant n$.

DEFINITION 3.1. The bracket ring $B_{G}$ of $G(S)$ is the ring $R_{G} / I_{G}$.

REMARK 3.2. We refer to the elements of $I_{G}$ of the above four types, as well as the equations formed by setting them equal to zero, as relations; in particular (3) are antisymmetry relations and (4) are the multiple syzygies, or simply syzygies. These relations are abstracted from the properties of determinants, with the syzygies in particular corresponding to Laplace's expansion by minors (Greene [2]). Indeed, if we regard $\left[x_{1}, \cdots, x_{n}\right]$ as an arbitrary determinant with columns $\left\{x_{1}, \cdots, x_{n}\right\}$ and $\left\{y_{1}, \cdots, y_{n}\right\}$ as the columns of the identity matrix, then the right side of (4) is the expansion of $\left[x_{1}, \cdots, x_{n}\right]$ by the first $k$ columns.

The ordinary syzygies, or syzygies with $k=1$, were studied in [13]. In the case of ordinary syzygies, if $\left\{x_{1}, \cdots, x_{n}\right\}$ and $\left\{y_{1}, \cdots, y_{n}\right\}$ are bases of $G(S)$, then by the simultaneous exchange axiom, one of the terms on the right of (4) involves bases in both brackets. This property carries over to the multiple syzygies in the following proposition, proved by Greene [2].

Proposition 3.3 (Multiple Exchange Property). If $G(S)$ is any pregeometry with bases $B$, and if $A, B \in B$ and $D \subseteq B$, then there exists $C \subseteq A$ such that $(A-C) \cup D \in B$ and $(B-D) \cup C \in B$. 
If $[X]$ is a bracket in $R_{G}, J$ an ideal of $R_{G}$, and $\alpha: R_{G} \rightarrow R_{G} / J$ the canonical homomorphism, we denote $\alpha([X])$ simply by $[X]$, where confusion will not arise. We assume that $1 \mapsto 1$ for all ring homomorphisms.

4. Coordinatizations. Let $V$ be a vector space of dimension $n$ over a field $K$, and $G(S)$ a combinatorial pregeometry of rank $n$. A coordinatization of $G(S)$ in $V$ is a mapping $\zeta: S \rightarrow V$ such that, for all $A \subseteq S, A$ is independent in $G$ if and only if $\zeta(A)$ is linearly independent in $\bar{V}$ and $\zeta$ is oneto-one on $A$. If $U: V \rightarrow V$ is a unimodular linear transformation, we say that $\zeta$ and $U \circ \zeta$ are equivalent coordinatizations. Given $\zeta$, we define a ring homomorphism $\zeta^{*}: R_{G} \rightarrow K$ by

$$
\zeta^{*}\left(\left[x_{1}, \cdots, x_{n}\right]\right)=\operatorname{det}\left(\zeta\left(x_{1}\right), \cdots, \zeta\left(x_{n}\right)\right),
$$

where determinants are defined with respect to a fixed basis of $V$.

Proposition 4.1. $\zeta^{*}$ factors through a homomorphism $\zeta^{* *}: B_{G} \rightarrow K$. Equivalent coordinatizations $\zeta$ and $U \circ \zeta$ determine the same homomorphism $\zeta^{*}$.

Proof. $I_{G} \subseteq \operatorname{ker} \zeta^{*}$, since relations (1), (2), and (3) are mapped by $\zeta^{*}$ to well-known relations on determinants, and Greene [2] proves that (4) similarly holds for determinants. Thus $\zeta^{* *}$ is defined. The second assertion is obvious.

A three-term syzygy is an element of $I_{G}$ of the form

$$
\begin{array}{r}
{\left[x_{1}, \cdots, x_{n}\right]\left[y_{1}, y_{2}, x_{3}, \cdots, x_{n}\right]-\left[y_{1}, x_{2}, \cdots, x_{n}\right]\left[x_{1}, y_{2}, x_{3}, \cdots, x_{n}\right]} \\
-\left[y_{2}, x_{2}, \cdots, x_{n}\right]\left[y_{1}, x_{1}, x_{3}, \cdots, x_{n}\right],
\end{array}
$$

where the remaining terms of the syzygy have been omitted by (1). Let $I_{G}^{\prime \prime}$ be the ideal generated by (1), (2), (3) and the three-term syzygies.

Proposition 4.2. Let $\nu: R_{G} \rightarrow K$ be a ring homomorphism into the field $K$ such that $\nu([X]) \neq 0$ if $X$ is a basis of $G$, and such that $I_{G}^{\prime \prime} \subseteq$ ker $\nu$. Then there is a coordinatization $\zeta$ of $G$ in a vector space $V$ of dimension $n$ over $K$, unique up to equivalence, such that $\nu=\zeta^{*}$.

Proof. Let $Y=\left\{y_{1}, \cdots, y_{n}\right\}$ be any prescribed basis of $G$, whence $\nu([Y]) \neq 0$. We define a new homomorphism $\mu: R_{G} \rightarrow K$ given by $\mu([X])=$ $v([X]) / \nu([Y])$. We note $I_{G}^{\prime \prime} \subseteq \operatorname{ker} \mu$ and $\mu([Y])=1$.

We proceed to define $\zeta: S \rightarrow V$ by letting $\zeta(x)^{(i)}$, the $i$ th component of $\zeta(x)$, be $\mu\left(\left[y_{1}, \cdots, y_{n} / y_{i} \rightarrow x\right]\right)$.

We assert that $\mu([X])=\operatorname{det}(\zeta X)$ for any bracket $[X]$. If $X$ has repeated elements, then $\mu([X])=\operatorname{det}(\zeta X)=0$. Assuming otherwise, we shall prove the assertion by induction on $k(X)=|X-Y|$. 
If $k(X)=0$ or 1 , then $\mu([X])=\operatorname{det}(\zeta X)$ by construction. Let $l \geqslant 2$ and suppose the assertion is true for $k(X)<l$. Let $Z=\left\{z_{1}, \cdots, z_{n}\right\}$ and $k(Z)=l$.

Case I. Suppose $r(Z) \geqslant n-2$ in $G(S)$. Then we may choose an independent subset $Z-\left\{z_{p}, z_{q}\right\}$ of cardinality $n-2$ with $z_{p}, z_{q} \notin Y$, by extending the independent subset $Z \cap Y$. Since $Y$ is a basis, by basis exchange we may extend $Z-\left\{z_{p}, z_{q}\right\}$ to a basis $W=\left(Z-\left\{z_{p}, z_{q}\right\}\right) \cup\left\{y_{r}, y_{s}\right\}$, where $y_{r}, y_{s} \in$ $Y$. Since antisymmetry applies to both brackets and determinants, we may assume $Z$ is reordered so that $z_{p}, z_{q}$ come first, and we let $U$ denote the remaining elements in order. Let $W$ have the ordering $y_{r}, y_{s}, U$.

Applying a three-term syzygy,

$$
\mu([Z]) \mu([W])=\mu\left(\left[y_{r}, z_{q}, U\right]\right) \mu\left(\left[z_{p}, y_{s}, U\right]\right)+\mu\left(\left[z_{p}, y_{r} U\right]\right) \mu\left(\left[z_{q}, y_{s}, U\right]\right) .
$$

Except for $[Z]$, each bracket here has $k \leqslant l-1$. Furthermore, $\mu([W]) \neq 0$. Thus we may solve for $\mu([Z])$ and conclude $\mu([Z])=\operatorname{det}(\zeta Z)$, since the determinants must satisfy the analogous syzygy.

Case II. Suppose $r(Z)<n-2$ in $G(S)$, whence $\mu([Z])=0$. Suppose further that $\operatorname{det}(\zeta Z) \neq 0$. Then $\left\{\zeta z_{1}, \cdots, \zeta z_{n}\right\}$ is a basis of $V$. Choose $z_{i} \notin Y, i \leqslant n$. Then, by basis exchange, $\left\{\zeta z_{1}, \cdots, \zeta z_{n} / \zeta z_{i} \rightarrow \zeta y_{j}\right\}$ is a basis of $V$ for some $y_{j} \in Y$. If $X=\left\{z_{1}, \cdots, z_{n} / z_{i} \rightarrow y_{j}\right\}, k(X)=l-1$, hence $\mu([X])=\operatorname{det} \zeta X \neq 0$. Hence $X$ is a basis of $G$, and $Z-\left\{z_{i}\right\}=X-\left\{y_{j}\right\}$ is of rank $n-1$, contradicting the assumption that $r(Z)<n-2$. Hence $\operatorname{det}(\zeta Z)=$ 0 as required for Case II.

We may now multiply the first coordinate of every vector $\zeta(x)$ by $v([Y])$ to reverse the normalization at the beginning of the proof, and the required coordinatization has been constructed. It is straightforward to check the uniqueness up to equivalence, completing the proof.

Let $G(S)$ and $F(S)$ be pregeometries on the same set $S$, and suppose that

(i) $\operatorname{rank} G=\operatorname{rank} F$,

(ii) $A$ is dependent in $G \Rightarrow A$ is dependent in $F$, for all $A \subseteq S$; equivalently, every basis of $F$ is a basis of $G$.

Then we say that $F$ is a rank-preserving-weak-map image of $G$. These images have been studied extensively in [5].

THEOREM 4.3. Let $G(S)$ be a combinatorial pregeometry and $K$ a field. There is a canonical bijection between the set of all homomorphisms $\eta: B_{G} \rightarrow K$ such that $[X] \notin \operatorname{ker} \eta$ for some $X \subseteq S$ and the set of all equivalence classes of coordinatizations over $K$ of the rank-preserving-weak-map images of $G$.

Proof. Let $\zeta$ be a coordinatization of $F$, where $F$ is a rank-preservingweak-map image of $G$. Then $R_{G}=R_{F}$ and $I_{F} \supseteq I_{G}$, thus we have the canonical 
homomorphism $\pi: B_{G} \rightarrow B_{F}$. Let $\eta=\zeta^{* *} \bullet \pi: B_{G} \rightarrow K$. We note that $\eta$ is nonzero on $[X]$ whenever $X$ is a basis of $F$, hence $\eta$ is the required homomorphism.

Conversely, let $\eta: B_{G} \rightarrow K$ be a homomorphism with $\eta([X]) \neq 0$ for some $X \subseteq S$. We define a combinatorial pregeometry $F(S)$ by letting the bases of $F$ be the collection

$$
B=\left\{\left\{x_{1}, \cdots, x_{n}\right\}: \eta\left(\left[x_{1}, \cdots, x_{n}\right]\right) \neq 0\right\} .
$$

The collection $B$ is nonempty, and the basis exchange property holds in $B$ since $\eta$ maps any syzygy to 0 . Thus $F$ is indeed a combinatorial pregeometry, and a rank-preserving-weak-map image of $G$, since any basis of $F$ is a basis of $G$.

We now note that $\eta$ must factor through a homomorphism $\nu: B_{F} \rightarrow K$, where $\eta=\nu \cdot \bullet \pi$ and $\nu\left(\left[x_{1}, \cdots, x_{n}\right]\right) \neq 0$ whenever $\left\{x_{1}, \cdots, x_{n}\right\}$ is a basis of $F$. Since $I_{F}^{\prime \prime} \subseteq I_{F}$, Proposition 4.2 gives the required equivalence class of coordinatizations of $F$.

It is clear that the two mappings we have constructed between the set of homomorphisms and the set of equivalence classes of coordinatizations are inverse to each other, completing the proof of Theorem 4.3.

Corollary 4.4. To every prime ideal $P \subseteq B_{G}$ such that $[X] \notin P$ whenever $[X] \neq 0$ in $B_{G}$, there corresponds a coordinatization of $G$ over $K_{P}$, the field of fractions of $B_{G} / P$, or over any extension field $K$ of $K_{P}$. Conversely, any coordinatization corresponds uniquely to such a prime ideal $P$.

Proof. We construct the canonical homomorphism $B_{G} \rightarrow B_{G} / P . \hookrightarrow K_{P} \hookrightarrow$ $K$. Conversely, given a coordinatization, let $P$ be the kernel of the corresponding homomorphism.

Corollary 4.5. Let $I_{G}^{\prime}$ denote the ideal in $R_{G}$ generated by (1), (2), (3) and the ordinary syzygies. Then $I_{G}^{\prime \prime} \subseteq I_{G}^{\prime} \subseteq I_{G}$, and $\operatorname{rad} I_{G}^{\prime \prime}=\operatorname{rad} I_{G}^{\prime}=$ $\operatorname{rad} I_{G}$.

Proof. The containments are obvious. If $P \supseteq I_{G}^{\prime \prime}$ is a prime ideal in $R_{G}$, then $P \supseteq I_{G}$ by 4.2 and 4.3. Thus $I_{G} \subseteq \operatorname{rad} I_{G}^{\prime \prime}=\bigcap\left\{P: P\right.$ is prime, $\left.P \supseteq I_{G}^{\prime \prime}\right\}$, and the rest follows.

The following proposition is closely related to a result of Vamos [11].

Proposition 4.6. Suppose that $[X][Y] \cdots[Z] \notin I_{G}^{\prime \prime}$ in $R_{G}$ whenever $X, Y, \cdots, Z$ are bases of $G$. Then $G$ may be coordinatized over some field.

Proof. Let $Q$ denote the multiplicative semigroup generated by the brackets on bases in $R_{G}$. Our hypothesis is that $Q \cap I_{G}^{\prime \prime}=\varnothing$. Standard ring theory arguments provide an ideal $P$ which is maximal in $\{J: J$ is an ideal of 
$\left.R_{G}, J \cap Q=\varnothing, J \supseteq I_{G}^{\prime \prime}\right\}$, and $P$ must be prime (as in [17, p. 152]). Then $P \supseteq I_{G}$, and $P / I_{G}$ is a prime ideal in $B_{G}$ which corresponds to the required coordinatization.

COROllary 4.7. There exist bases $X, Y, \cdots, Z$ in $G$ such that $[X][Y] \cdots[Z]=0$ in $B_{G}$ if and only if $G$ may not be coordinatized over any field.

REMARK 4.8. In [14], the sequel, we explore the following converse: if $G$ is unimodular (i.e., $G$ is coordinatizable over the rationals by a totally unimodular matrix), then $B_{G}$ is an integral domain.

5. The bracket representation. We now represent any combinatorial pregeometry $G(S)$ in a module over its own bracket ring. This representation is rather crude, since independent sets may become dependent under the representation, and since the representation depends heavily on a choice of basis. However, we receive compensation in that the representation works for an arbitrary pregeometry, and it allows a pleasing imitation of several familiar results from linear algebra, with the brackets replacing scalars.

Let $E=\left\{e_{1}, \cdots, e_{n}\right\}$ be a fixed basis of $G(S)$. Let $M=\bigoplus_{i=1}^{n} B_{G} \cdot e_{i}$ be the free module over $B_{G}$ generated by the symbols $e_{1}, \cdots, e_{n}$. We define a mapping $\gamma: S \rightarrow M$ as follows.

Definition 5.1 (CRAMER'S RUle). $\gamma: x \mapsto \Sigma_{i=1}^{n}\left[e_{1}, \cdots, e_{n} / e_{i} \rightarrow x\right] e_{i}$.

THEOREM 5.2. $\gamma$ maps dependent subsets of $G(S)$ onto linearly dependent subsets of $M$.

Proof. It suffices to show that $\gamma(C)$ is linearly dependent, where $C=$ $\left\{c_{1}, \cdots, c_{k}\right\}$ is a circuit of $G$. We extend the independent set $C-\left\{c_{1}\right\}$ to a basis $\left(C-\left\{c_{1}\right\}\right) \cup A$, where $A \cap\left(C-\left\{c_{1}\right\}\right)=\varnothing$. Then

$$
\begin{aligned}
& \sum_{j=1}^{k}(-1)^{j}\left[c_{1}, \cdots, c_{j-1}, c_{j+1}, \cdots, c_{k}, A\right] \gamma\left(c_{j}\right) \\
& =\sum_{i=1}^{n}\left(\sum_{j=1}^{k}(-1)^{j}\left[c_{1}, \cdots, c_{j-1}, c_{j+1}, \cdots, c_{k}, A\right]\left[e_{1}, \cdots, e_{n} / e_{i} \rightarrow c_{j}\right]\right) e_{i} \\
& =\sum_{i=1}^{n}\left(-\left[c_{2}, \cdots, c_{k}, A\right]\left[e_{1}, \cdots, e_{n} / e_{i} \rightarrow c_{1}\right]\right. \\
& \left.\quad+\sum_{j=2}^{k}\left[c_{2}, \cdots, c_{k}, A / c_{j} \rightarrow c_{1}\right]\left[e_{1}, \cdots, e_{n} / e_{i} \rightarrow c_{j}\right]\right) e_{i} \\
& =0 .
\end{aligned}
$$


Thus $\gamma(C)$ is linearly dependent, since the coefficient of $\gamma\left(c_{1}\right)$ (and, in fact, of each $\gamma\left(c_{j}\right)$ ) is nonzero in $B_{G}$.

We now extend $M$ to $M^{\prime}=\bigoplus_{D: \phi \neq D \subseteq E} B_{G} \cdot D$, and extend $\gamma$ to $\gamma^{\prime}: I \rightarrow M^{\prime}$, where $I$ is the collection of ordered independent sets in $G(S)$, with $\gamma^{\prime}$ defined by

$$
\begin{aligned}
& \gamma^{\prime}\left(x_{1}, \cdots, x_{k}\right) \\
& \quad=\sum_{i_{1}<\ldots<i_{k}}\left[e_{1}, \cdots, e_{n} / e_{i_{1}} \rightarrow x_{1}, \cdots, e_{i_{k}} \rightarrow x_{k}\right] \cdot\left\{e_{i_{1}}, \cdots, e_{i_{k}}\right\}
\end{aligned}
$$

for any $\left(x_{1}, \cdots, x_{k}\right) \in I$.

Under any coordinatization $\zeta,\left[e_{1}, \cdots, e_{n} / e_{i_{1}} \rightarrow x_{1}, \cdots, e_{i_{k}} \rightarrow x_{k}\right]$ corresponds to the $\left(i_{1}, \cdots, i_{k}\right)$ th Plucker coordinate of the subspace spanned by $\left\{\zeta\left(x_{1}\right), \cdots, \zeta\left(x_{k}\right)\right\}$, with respect to the base $\zeta\left(e_{1}\right), \cdots, \zeta\left(e_{n}\right)$. The following proposition shows that the coefficients $\left[e_{1}, \cdots, e_{n} / e_{i_{1}} \rightarrow x_{1}, \cdots, e_{i_{k}} \rightarrow\right.$ $\left.x_{k}\right]$ depend only on the flat $\mathrm{Cl}\left\{x_{1}, \cdots, x_{k}\right\}$, up to a scalar multiple, hence these coefficients may be regarded as the Plucker coordinates of the flat $\mathrm{Cl}\left\{x_{1}, \cdots, x_{k}\right\}$ of $G(S)$, with respect to the basis $\left\{e_{1}, \cdots, e_{n}\right\}$.

Proposition 5.3. If $\left(x_{1}, \cdots, x_{k}\right)$ and $\left(y_{1}, \cdots, y_{k}\right) \in I$, and $\mathrm{Cl}\left\{x_{1}, \cdots, x_{k}\right\}=\mathrm{Cl}\left\{y_{1}, \cdots, y_{k}\right\}$ in $G(S)$, then there exist nonzero elements $a, b \in B_{G}$ such that

$$
\begin{aligned}
a\left[e_{1}, \cdots, e_{n} / e_{i_{1}} \rightarrow\right. & \left.x_{i}, \cdots, e_{i_{k}} \rightarrow x_{k}\right] \\
& =b\left[e_{1}, \cdots, e_{n} / e_{i_{1}} \rightarrow y_{1}, \cdots, e_{i_{k}} \rightarrow y_{k}\right]
\end{aligned}
$$

for all $i_{1}<\cdots<i_{k}$.

Proof. By the multiple exchange property, Proposition 3.3, there exists $\left(j_{1}, \cdots, j_{k}\right)$ such that $\left[e_{1}, \cdots, e_{n} / e_{j_{1}} \rightarrow x_{1}, \cdots, e_{j_{k}} \rightarrow x_{k}\right] \neq 0$. Let this bracket be $b$, and $a=\left[e_{1}, \cdots, e_{n} / e_{j_{1}} \rightarrow y_{1}, \cdots, e_{j_{k}} \rightarrow y_{k}\right]$. Since $\mathrm{Cl}\left\{x_{1}, \cdots, x_{k}\right\}=\mathrm{Cl}\left\{y_{1}, \cdots, y_{k}\right\}, b \neq 0$ implies $a \neq 0$. We now have a syzygy

$$
\begin{aligned}
& {\left[e_{1}, \cdots, e_{n} / e_{j_{1}} \rightarrow y_{1}, \cdots, e_{j_{k}} \rightarrow y_{k}\right]\left[e_{1}, \cdots, e_{n} / e_{i_{1}} \rightarrow x_{1}, \cdots, e_{i_{k}} \rightarrow x_{k}\right]} \\
& \quad=\left[e_{1}, \cdots, e_{n} / e_{j_{1}} \rightarrow x_{1}, \cdots, e_{j_{k}} \rightarrow x_{k}\right]\left[e_{1}, \cdots, e_{n} / e_{i_{1}} \rightarrow y_{1}, \cdots, e_{i_{k}} \rightarrow y_{k}\right],
\end{aligned}
$$

for $\left\{y_{1}, \cdots, y_{k}\right\}$ of the first bracket may be exchanged only for $\left\{x_{1}, \cdots, x_{k}\right\}$ in the second to give two bases. All other terms of the syzygy have a bracket 
containing at least $k+1$ of $\left\{x_{1}, \cdots, x_{k}, y_{1}, \cdots, y_{k}\right\}$, hence containing a dependent set. The above syzygy is precisely what is required.

6. An identity of van der Waerden. We now derive an alternate form of the syzygies, which is useful in the next section. This derivation also clarifies the relationship between the multiple and ordinary syzygies.

Let $\left[X_{1}, A_{1}\right] \cdots\left[X_{p}, A_{p}\right] \in R_{G}$, where $X_{1}, \cdots, X_{p}$ are pairwise disjoint and have distinct elements. Let $T=X_{1} \cup \cdots \cup X_{p}, j_{i}=\left|X_{i}\right|, \quad m=$ $\Sigma_{i=1}^{p} j_{i}=|T|$. In [12, Hilfssatz 1, p. 723], van der Waerden proved the following identity, provided $m \geqslant n+1$.

$$
\sum(\operatorname{sgn} \sigma)\left[\sigma X_{1}, A_{1}\right] \cdots\left[\sigma X_{p}, A_{p}\right] \in I_{G}^{\prime},
$$

where the sum is over all permutations $\sigma$ of $T$, and $I_{G}^{\prime}$ is the ideal of ordinary syzygies defined in Corollary 4.5. However, there is considerable redundancy built into this identity, for if $\tau$ is any permutation of $T$ fixing the subsets $\sigma X_{1}$, $\cdots, \sigma X_{p}$, then the summand for $\tau \sigma$ is the same as that for $\sigma$, by antisymmetry.

DEFinition 6.1. A van der Waerden relation is an element of $R_{G}$ of the form

$$
W\left(j_{1}, \cdots, j_{p} ; T ; A_{1}, \cdots, A_{p}\right)=\sum(\operatorname{sgn} \sigma)\left[T_{1}, A_{1}\right] \cdots\left[T_{p}, A_{p}\right],
$$

where the sum is over all partitions $T=T_{1} \cup \cdots \cup T_{p}$ with $\left|T_{i}\right|=j_{i}$, and where $\operatorname{sgn} \sigma$ is chosen as in (5) according to the order in which we write the elements in each $T_{i}$. We include $j_{1}, \cdots, j_{p}$ as arguments of $W$ for convenience, in spite of the redundancy. Now, if $m \geqslant n+1$, (5) becomes

$$
\left(\prod_{i=1}^{p} j_{i} !\right) W\left(j_{1}, \cdots, j_{p} ; T ; A_{1}, \cdots, A_{k}\right) \in I_{G}^{\prime} .
$$

We now wish to eliminate the numerical factor and generalize to a larger class of sets $T$, which is made possible by using the larger ideal $I_{G}$.

THEOREM 6.2. Let $T$ be dependent in $G$. Then

$$
W\left(j_{1}, \cdots, j_{p} ; T ; A_{1}, \cdots, A_{p}\right) \in I_{G} .
$$

Proof. We rearrange the order of the brackets so that $j_{1} \leqslant j_{2} \leqslant \cdots \leqslant j_{p}$.

Case 1. Suppose $j_{p}=n$ and $j_{1}=\cdots=j_{p-2}=0$. If we also have $j_{p-1}=0$ we are done, for $W$ consists of one term, and $W \in I_{G}$ by the dependence of $T$. Otherwise, let $j=j_{p-1}, T=\left\{x_{1}, \cdots, x_{j}, y_{1}, \cdots, y_{n}\right\}, V=$ $\left\{x_{1}, \cdots, x_{j}\right\}$, and if $U \subseteq V, U \neq \varnothing$, we denote $U$ by $\left\{x_{m_{1}}, \cdots, x_{m_{l}}\right\}$. Then 


$$
\begin{aligned}
& w\left(0, \cdots, 0,1, n ; T ; A_{1}, \cdots, A_{p-1}, \varnothing\right) \\
& =\left[A_{1}\right] \cdots\left[A_{p-2}\right]\left(\left[x_{1}, \cdots, x_{p}, A_{p-1}\right]\left[y_{1}, \cdots, y_{n}\right]\right. \\
& +\sum_{U: \Phi \neq U \subseteq V} \sum_{i_{1}<\ldots<i_{l}}(-1)^{l}\left[x_{1}, \cdots, x_{i p}, A_{p-1} / x_{m_{1}} \rightarrow y_{i_{1}}, \cdots, x_{m_{l}} \rightarrow y_{i_{l}}\right] \\
& \left.\cdot\left[y_{1}, \cdots, y_{n} / y_{i_{1}} \rightarrow x_{m_{1}}, \cdots, y_{i_{l}} \rightarrow x_{m_{l}}\right]\right) \\
& =\left[A_{1}\right] \cdots\left[A_{p-2}\right] \sum_{U: \Phi \neq U \subseteq V}\left((-1)^{1-1}\left[x_{1}, \cdots, x_{p}, A_{p-1}\right]\left[y_{1}, \cdots, y_{n}\right]\right. \\
& +(-1)^{l} \sum_{i_{1}<\ldots<i_{l}}\left[x_{1}, \cdots, x_{i}, A_{p-1} / x_{m_{1}} \rightarrow y_{i_{1}}, \cdots, x_{m_{1}} \rightarrow y_{i_{l}}\right] \\
& \left.\cdot\left[y_{1}, \cdots, y_{n} / y_{i_{1}} \rightarrow x_{m_{1}}, \cdots, y_{i_{l}} \rightarrow x_{m_{l}}\right]\right) \text {. }
\end{aligned}
$$

hence $W \in I_{G}$.

Case 2. Suppose $j_{p}=n$ and $j_{p-2} \neq 0$. Then

$$
\begin{aligned}
& W\left(j_{1}, \cdots, j_{p-1}, n ; T ; A_{1}, \cdots, A_{p}\right) \\
& =\sum_{T_{1}, \cdots, T_{p-2}} W\left(0, \cdots, 0, j_{p-1}, n ; T-\bigcup_{i=1}^{p-2} T_{i} ;\right. \\
& \left.A_{1} \cup T_{1}, \cdots, A_{p-2} \cup T_{p-2}, A_{p-1}, A_{p}\right),
\end{aligned}
$$

where the sum is over pairwise disjoint subsets $T_{1}, \cdots, T_{p-2}$ with $\left|T_{i}\right|=j_{i}$, for $1 \leqslant i \leqslant p-2$. Since $j_{p-1}+j_{p} \geqslant n+1, T-\bigcup_{i=1}^{p-2} T_{i}$ is dependent, thus we are reduced to Case 1.

Case 3. Suppose $j_{p}<n$. Pick $a \in A_{p}$.

$$
\begin{aligned}
& W\left(j_{1}, \cdots, j_{p} ; T ; A_{1}, \cdots, A_{p}\right) \\
& \quad=W\left(j_{1}, \cdots, j_{p-1}, j_{p}+1 ; T \cup\{a\} ; A_{1}, \cdots, A_{p-1}, A_{p}-\{a\}\right) \\
& \quad-\sum_{i: 1<i<p-1 ; j_{i} \neq 0} W\left(j_{1}, \cdots, j_{i}-1, \cdots, j_{p-1}, j_{p}+1 ;\right.
\end{aligned}
$$

$$
\left.T ; A_{1}, \cdots, A_{i} \cup\{a\}, \cdots, A_{p-1}, A_{p}-\{a\}\right) .
$$

Since $j_{p}$ has been increased by unity, repeated use of the above reduces $W$ to a linear combination of $W^{\prime}$ s with $j_{p}=n$, each of which is covered by Case 1 or 2. Thus the proof of Theorem 6.2 is completed.

Let us denote, for any product of brackets $[X][Y]$ and $V \subseteq X$,

$$
S(V)=[X][Y]-\sum_{U \subseteq Y ;|U|=|V|} \pm[X-V, U][V, Y-U]
$$

where the signs are appropriately chosen so that $S(V)$ is a syzygy. Let $J_{G}$ 
denote the ideal in $R_{G}$ generated by the relations (1), (2), and (3).

LEMMA 6.3. $S(V)= \pm S(X-V)$ in $R_{G} / J_{G}$.

Proof. By applying the same permutation to each left-hand bracket in $S(V)$ (resp. $S(X-V)$ ), we may write

$$
\begin{aligned}
\pm S(V)=\left[v_{1}, \cdots, v_{k}, w_{k+1}, \cdots, w_{n}\right]\left[y_{1}, \cdots, y_{n}\right] \\
-\sum_{t_{1}<\ldots<i_{k}}\left[y_{i_{1}}, \cdots, y_{i_{k}}, w_{k+1}, \cdots, w_{n}\right] \\
\quad\left[y_{1}, \cdots, y_{n} / y_{i_{1}} \rightarrow v_{1}, \cdots, y_{i_{k}} \rightarrow v_{k}\right]
\end{aligned}
$$

and

$$
\pm S(X-V)=\left[v_{1}, \cdots, v_{k}, w_{k+1}, \cdots, w_{n}\right]\left[y_{1}, \cdots, y_{n}\right]
$$

$$
\begin{aligned}
-\sum_{j_{k+1}<\ldots<j_{n}}\left[v_{1}, \cdots, v_{k}, y_{j_{k+1}}, \cdots, y_{j_{n}}\right] & \\
& \cdot\left[y_{1}, \cdots, y_{n} / y_{j_{k+1}} \rightarrow w_{k+1}, \cdots, y_{j_{n}} \rightarrow w_{n}\right] .
\end{aligned}
$$

Let $\left(i_{1}, \cdots, i_{k}\right)$, where $i_{1}<\cdots<i_{k}$, correspond to $\left(j_{k+1}, \cdots, j_{n}\right)$, where $\left\{j_{k+1}, \cdots, j_{n}\right\}=\{1, \cdots, n\}-\left\{i_{1}, \cdots, i_{k}\right\}$ and $j_{k+1}<\cdots<j_{n}$. Then it is a routine exercise to check that the signs of corresponding terms on the right of (8) and (9) agree, hence $S(V)= \pm S(X-V)$.

For any product of two brackets $[X][Y]$ and $V \subseteq X$, let $W(V)=$ $W(|V|, n ; V \cup Y ; X-V, \varnothing)$.

Proposition 6.4. The ideal $I_{G}$ is generated by the relations (1), (2), (3) and the van der Waerden relations $W(V)$ for all $X, Y, V$ with $|V| \leqslant n / 2$.

Proof. We have from (7), $W(V)=\Sigma_{U \subseteq V}(-1)^{|U|-1} S(U)$. By Möbius inversion (see [7, pp. 344-345]) over the lattice of subsets of $V$, we have

$$
S(V)=(-1)^{|V|-1} \sum_{U \subseteq V}(-1)^{|V-U|} W(U)=\sum_{U \subseteq V}(-1)^{|U|-1} W(U) .
$$

Thus the van der Waerden relations may replace the syzygies as generators of $I_{G}$. By Lemma 6.3, we may assume $|V| \leqslant n / 2$.

COROLLARY 6.5. Let $m=[n / 2] ! n !$, where [] here denotes the greatest integer function. Then $m I_{G} \subseteq I_{G}^{\prime}$.

Proof. If $|V| \leqslant n / 2, m W(V) \in I_{G}^{\prime}$ by (6). Thus the result follows from the preceding proposition. 
7. Standard products. In this section we attempt to put each element of $B_{G}$ into a standard form, namely a linear combination of standard products of brackets, using the well-known techniques of Young tableaux ([16] and [3, pp. 377-380]). Unfortunately, this form is not unique except for a small class of pregeometries. Nevertheless, we gain insight into the structure of the bracket ring.

A multiset $M$ on the set $S$ is a formal linear combination of elements of $S$ with nonnegative integral coefficients. We denote $M=\Sigma_{s \in S} a_{s} \cdot s$, where $a_{s}$ is the multiplicity of $s$ in $M$, and $\Sigma_{s \in S} a_{s}$, if finite, is the size of $M$. We define addition of multisets in the obvious manner and note that the collection of all multisets on $S$ whose size is a finite multiple of $n$ form a commutative monoid $M_{n}$ under this addition operation.

We say a product of brackets $L=\left[X_{1}\right] \cdots\left[X_{l}\right]$ in $R_{G}$ or $B_{G}$ is of degree $M \in M_{n}$ if $M=\Sigma_{s \in S} a_{s} \cdot s$ where $a_{s}$ is the total number of occurrences of $s$ in $X_{1}, \cdots, X_{l}$.

Proposition 7.1. The rings $R_{G}$ and $B_{G}$ are graded rings over the commutative monoid $M_{n}$, which facts we denote by $R_{G}=\bigoplus_{M \in M_{n}} R_{G}(M)$ and $B_{G}=\bigoplus_{M \in M_{n}} B_{G}(M)$.

Proof. As an additive group, we clearly have the direct sum $R_{G}=$ $\bigoplus_{M \in M_{n}} R_{G}(M)$, where $R_{G}(M)$ is the subgroup of $R_{G}$ generated by all products of brackets of degree $M$. If $K_{1} \in R_{G}\left(M_{1}\right)$ and $K_{2} \in R_{G}\left(M_{2}\right)$ then $K_{1} K_{2} \in$ $R_{G}\left(M_{1}+M_{2}\right)$, hence $R_{G}$ is a graded ring over $M_{n}$. Since $I_{G}$ is generated by homogeneous elements, it is a homogeneous ideal, hence $B_{G}$ is also graded over $M_{n}$, with $B_{G}(M)$ defined analogously to $R_{G}(M)$.

We now consider any nonzero product of brackets $L=\left[X_{1}\right]\left[X_{2}\right] \cdots\left[X_{p}\right]$ of degree $M \in M_{n}$. Let $S$ be given a fixed linear ordering. By at most changing the sign of $L$, we may assume that the elements in each bracket in $L$ are written in increasing order, $\left[X_{i}\right]=\left[x_{i 1}, \cdots, x_{i n}\right]$, and that $X_{1}, X_{2}, \cdots, X_{p}$ are in lexicographic order. If $x_{1 j} \leqslant x_{2 j} \leqslant \cdots \leqslant x_{p j}$ for each $j$, we șay that $L$ is a standard product of brackets.

Proposition 7.2. The standard products of brackets of degree $M$ generate $B_{G}(M)$ as an additive group.

Proof. Let St $(M)$ be the additive subgroup of $B_{G}(M)$ generated by the standard products of brackets of degree $M$. It suffices to show that if $L$ is any product of brackets of degree $M$, then $L \in \mathrm{St}(M)$. If $L$ is not standard, let $(k, l)$ be the lexicographically least pair $(i, j)$ such that $x_{i j}<x_{(i-1) j}$. Thus $x_{i j} \geqslant x_{(i-1) j}$ for all $(i, j)$ such that $2 \leqslant i<k$ or $2 \leqslant i=k$ and $j<l$. If $L$ is standard, set $(k, l)=(p, n+1)$. We now proceed by reversed induction on $(k, l)$ in the lexicographic ordering, $(2,1),(2,2), \cdots,(2, n),(3,1), \cdots$, 
$(p, n),(p, n+1)$. If $(k, l)=(p, n+1)$, then $L \in \mathrm{St}(M)$. Otherwise, we assume by the induction hypothesis that if $L^{\prime}$ is of degree $M$ with $\left(k^{\prime}, l^{\prime}\right)$ defined for $L^{\prime}$ analogously to $(k, l)$ for $L$, and if $\left(k^{\prime}, l^{\prime}\right)>(k, l)$ in the lexicographic ordering, then $L^{\prime} \in \operatorname{St}(M)$.

We now apply Theorem 6.2 to $L$ with

$$
T=\left\{x_{k 1}, x_{k 2}, \cdots, x_{k l}, x_{(k-1) l}, \cdots, x_{(k-1) n}\right\} .
$$

Writing $L=K\left[X_{k-1}\right]\left[X_{k}\right]$, we have

$$
\begin{array}{r}
L=-\sum(\operatorname{sgn} \sigma) K\left[x_{(k-1) 1}, \cdots, x_{(k-1)(l-1)}, T-T^{\prime}\right] \\
\cdot\left[T^{\prime}, x_{k(l+1)}, \cdots, x_{k n}\right]
\end{array}
$$

where the sum is over all $T^{\prime} \subseteq T$ such that $\left|T^{\prime}\right|=l, T^{\prime} \neq\left\{x_{k 1}, \cdots, x_{k l}\right\}$. Since $x_{k 1}<\cdots<x_{k l}<x_{(k-1) l}<\cdots<x_{(k-1) n}$ and $x_{(k-1) j}<x_{k j}$ for all $j<l$, we see, after arranging the elements of $T^{\prime}$ and $T-T^{\prime}$ in increasing order, that each summand on the right of $(10)$ is a product $L^{\prime}$ with $\left(k^{\prime}, l^{\prime}\right)>(k, l)$ in the lexicographic ordering. It follows from the induction hypothesis that $L \in$ St $(M)$, as required.

We now proceed to investigate the class of pregeometries for which the standard products are actually a basis of $B_{G}(M)$.

Proposition 7.3. Let $G(S)$ be the free pregeometry on a set $S$ of cardinality $N$ whose bases are all $n$-subsets of $S$. Then the standard products of degree $M$ are a linear basis of $B_{G}(M)$ over the integers, for all $M \in M_{n}$.

Proof. Let $\mathbf{Q}$ denote the rationals, and let $S=\left\{s_{1}, \cdots, s_{N}\right\}$. We construct a coordinatization $\zeta$ of $G(S)$ over $Q\left(t, x_{i j}: 1 \leqslant i \leqslant N, 1 \leqslant j \leqslant n\right)$, where $t, x_{11}, \cdots, x_{N n}$ are algebraically independent transcendentals over $Q$. The coordinatization is given by

$$
\zeta: s_{i} \mapsto\left(x_{i j} t^{(N-i)(N-i+j)}\right)_{j=1}^{N} .
$$

.An argument of Hodge and Pedoe [3, p. 381] now shows that the polynomials which are the determinants corresponding under $\zeta^{* *}$ to the standard products of brackets are linearly independent for any $M$, hence the standard products of brackets are linearly independent in $B_{G}(M)$.

A transversal geometry is a geometry $G(S)$ which may be coordinatized over $\mathrm{Q}\left(x_{i j}\right)$ by $s_{i} \mapsto\left(y_{i j}\right)$, where the $x_{i j}$ are algebraically independent transcendentals over $Q$ and $y_{i j}=x_{i j}$ or 0 . By Mirsky [6, Chapter 6.5], this definition is equivalent to the usual one in terms of a bipartite graph. The preceding proof suggest the following conjecture, which has withstood attempts to find a counterexample. 
Conjecture 7.4. If $G$ is a transversal geometry, then for each $M \in M_{n}$, there exists a linear ordering on $S$ such that the nonzero standard products of brackets in $B_{G}(M)$ are a basis of $B_{G}(M)$.

8. The geometric structure of the bracket ring. Several important geometric properties are reflected in the structure of the bracket ring. In particular, orthogonal pregeometries have isomorphic bracket rings, and the bracket rings of minors of $G$ are contained (up to isomorphism) in the bracket ring of $G$.

If $G(S)$ is a finite pregeometry with bases $B$, the pregeometry $G^{*}(S)$ orthogonal to $G$ has bases $B^{*}=\{S-B: B \in B\}$. Thus $G$ is also orthogonal to $G^{*}$, and we say $G$ and $G^{*}$ are dual.

THEOREM 8.1. Let $G(S)$ and $G^{*}(S)$ be dual pregeometries. Then $B_{G} \cong B_{G} *$.

Proof. We fix a linear ordering $L=\left(s_{1}, \cdots, s_{N}\right)$ on $S, S=\left\{s_{1}, s_{2}\right.$, $\left.\cdots, s_{N}\right\}$. We define a ring homomorphism $h_{L}: R_{G} \rightarrow B_{G} *$ by

(i) $h_{L}([X])=0$ if $X$ has repeated elements,

(ii) $h_{L}\left(\left[x_{1}, \cdots, x_{n}\right]\right)=\operatorname{sgn} \rho\left[s_{\rho(n+1)}, \cdots, s_{\rho(N)}\right]$, if $x_{1}, \cdots, x_{n}$ are distinct, where $\rho$ is any permutation of $\{1, \cdots, N\}$ such that $x_{i}=s_{\rho(i)}$ for all $i, 1 \leqslant i \leqslant n$,

and $h_{L}$ is extended to any element of $R_{G}$ in the obvious manner.

We note first that $h_{L}$ is well defined, for the antisymmetry in $B_{G}$ * implies that $h_{L}\left(\left[x_{1}, \cdots, x_{n}\right]\right)$ in (ii) does not depend on the choice of $\rho$.

We now check that $h_{L}(Q)=0$ if $Q$ is any of the generators (1)-(4) of $I_{G}$. If $Q=[X]$ where $X$ has repeated elements, then $h_{L}(Q)=0$ by definition. If $Q=[X]$ where the elements of $X$ are distinct but dependent, then $h_{L}(Q)=[S-X]$ for some ordering of $S-X$. Since $X$ is not a basis of $G$, $S-X$ is not a basis of $G^{*}$, and $h_{L}(Q)=0$. If $Q=[X]-\operatorname{sgn} \sigma[\sigma X]$, where $X=\left(x_{1}, \cdots, x_{n}\right)$ has distinct elements, then if $\rho$ is prescribed as in (ii) for $X$, then $\sigma \rho$ satisfies (ii) for $\sigma X$, where $\left.\sigma \rho\right|_{S-X}=\left.\rho\right|_{S-X}$, and

$$
\begin{aligned}
h_{L}(Q) & =h_{L}([X])-\operatorname{sgn} \sigma h_{L}([\sigma X]) \\
& =\operatorname{sgn} \rho\left[x_{\rho(n+1)}, \cdots, x_{\rho(N)}\right]-\operatorname{sgn} \sigma \operatorname{sgn} \sigma \rho\left[x_{\rho(n+1)}, \cdots, x_{\rho(N)}\right] \\
& =0 .
\end{aligned}
$$

We have yet to check generators of type (4). Let

$$
\begin{aligned}
& Q=\left[x_{1}, \cdots, x_{n}\right]\left[y_{1}, \cdots, y_{n}\right] \\
&-\sum_{1<i_{1}<i_{2}<\ldots<i_{k}<n}\left[x_{1}, \cdots, x_{n} / x_{i_{1}} \rightarrow y_{1}, \cdots, x_{i_{k}} \rightarrow y_{k}\right] \\
& \quad \cdot\left[x_{i_{1}}, \cdots, x_{i_{k}}, y_{k+1}, \cdots, y_{n}\right] .
\end{aligned}
$$


Modulo the ideal $J_{G}$ generated by relations (1), (2), (3), which we know is contained in $\operatorname{ker} h_{L}$, we may write

$$
\begin{array}{r}
Q=\left[x_{1}, \cdots, x_{l}, y_{l+1}, \cdots, y_{n}\right]\left[y_{1}, \cdots, y_{n}\right] \\
-\sum_{1<i_{1}<i_{2}<\ldots<i_{j}<l}\left[x_{1}, \cdots, x_{l}, y_{l+1}, \cdots, y_{n} / x_{i_{1}} \rightarrow y_{1}, \cdots, x_{i_{j}} \rightarrow y_{j}\right] \\
\quad \cdot\left[x_{i_{1}}, \cdots, x_{i_{j}}, y_{j+1}, \cdots, y_{n}\right],
\end{array}
$$

where we have relabeled as $x_{l+1}, \cdots, x_{n}=y_{l+1}, \cdots, y_{n}$ (resp.) the elements common to both brackets, noting that any such common elements among $y_{1}$, $\cdots, y_{k}$ may be dropped from the summation. We now note that if $K$ is any ordering of $S$ affected by a permutation $\mu$ on $L$, then $h_{K}([X])=$ $(\operatorname{sgn} \mu) h_{L}([X])$ for any $X$, thus $h_{K}(Q)=h_{L}(Q)$ in the case of type (4) relations. We may therefore assume that $L$ is the ordering $\left(x_{1}, \cdots, x_{l}, y_{1}, \cdots\right.$, $\left.y_{n}, s_{n+l+1}, \cdots, s_{N}\right)$. We now compute:

$$
\begin{aligned}
h_{L}(Q)= & (-1)^{l(n-l)}\left[y_{1}, \cdots, y_{l}, s_{n+l+1}, \cdots, s_{N}\right] \\
& \cdot(-1)^{l n}\left[x_{1}, \cdots, x_{l}, s_{n+l+1}, \cdots, s_{N}\right] \\
- & \sum_{1<i_{1}<i_{2}<\cdots<i_{j}<l}(-1)^{j+l(n-l)}\left[x_{i_{1}}, \cdots, x_{i_{j}}, y_{j+1}, \cdots, y_{l}, s_{n+l+1}, \cdots, s_{N}\right] \\
& \cdot(-1)^{j+n l}\left[x_{1}, \cdots, x_{l}, s_{n+l+1}, \cdots, s_{N} / x_{i_{1}} \rightarrow y_{1}, \cdots, x_{i_{j}} \rightarrow y_{j}\right] \\
= & \text { in } B_{G^{*}} .
\end{aligned}
$$

Thus $\operatorname{ker} h_{L} \supseteq I_{G}$, and $h_{L}$ induces a homomorphism $j_{L}: B_{G} \rightarrow B_{G} *$. Dually, we have the homomorphism $k_{L}: B_{G} * \rightarrow B_{G}$ defined by the same linear ordering $L$. Let $\tau$ denote the permutation

$$
\left(\begin{array}{ccc}
1, & 2, \cdots, N-n, & N-n+1, \cdots, N \\
n+1, & n+2, \cdots, N, & 1, \cdots, n
\end{array}\right) .
$$

Then

$$
\begin{aligned}
k_{L} j_{L}\left[x_{1}, \cdots, x_{n}\right] & =(\operatorname{sgn} \rho) k_{L}\left[s_{\rho(n+1)}, \cdots, s_{\rho(N)}\right] \\
& =(\operatorname{sgn} \rho) k_{L}\left[s_{\rho \tau(1)}, \cdots, s_{\rho \tau(N-n)}\right] \\
& =(\operatorname{sgn} \rho)(\operatorname{sgn} \rho \tau)\left[s_{\rho \tau(N-n+1)}, \cdots, s_{\rho \tau(N)}\right] \\
& =\operatorname{sgn} \tau\left[x_{1}, \cdots, x_{n}\right] .
\end{aligned}
$$

Since $\operatorname{sgn} \tau=(-1)^{N(N-n)}$ is fixed, $k_{L} j_{L}$ is an automorphism of $B_{G}$, and 
similarly $j_{L} k_{L}$ is an automorphism of $B_{G} *$. Hence $j_{L}$ is an isomorphism, completing the proof of Theorem 8.1.

If $G(S)$ is a pregeometry with bases $B, U \subseteq S$, and $\operatorname{rank}(S-U)=$ rank $G$, then the subgeometry $G-U$ is the pregeometry on $S-U$ whose bases are $\{B: B \in B, B \subseteq S-U\}$. If $T \subseteq S$ is independent, the contraction $G / T$ is the pregeometry on $S-T$ whose bases are $\{B-T: B \in B, T \subseteq B\}$. We note that subgeometries are dual to contractions, $G^{*} / T=(G-T)^{*}$. A minor of $G$ is a subgeometry of a contraction, $(G / T)-U$, where $U \cap T=\varnothing$. (Although our definitions of subgeometry and contraction are more restrictive than those in Crapo-Rota [1], our definition of minor is equivalent to theirs.)

Given $T, U \subseteq S, T \cap U=\varnothing$, we define $B_{G}(T, U)$ to be the subring of $B_{G}$ generated by all brackets $[X]$ such that $X \supseteq T$ and $X \cap U=\varnothing$.

THEOREM 8.2. Let $G$ be a finite pregeometry and $F=(G / T)-U$ a minor of $G$. Then $B_{F} \cong B_{G}(T, U) \subseteq B_{G}$.

Proof. It is obvious that $B_{G-U} \cong B_{G}(\varnothing, U)$, and, similarly, that $B_{G-U}(T, \varnothing) \cong B_{G}(T, U)$. But $B_{G^{*}-T} \cong B_{G} *(\varnothing, T)$ implies by duality that $B_{G / T} \cong B_{G}(T, \varnothing)$. Thus $B_{F} \cong B_{G-U}(T, \varnothing) \cong B_{G}(T, U)$, since $(G / T)-U \cong$ $(G-U) / T$.

\section{REFERENCES}

1. H. Crapo and G.-C. Rota, Combinatorial geometries, M.I.T. Press, Cambridge, Mass., 1970 (preliminary edition). MR 45 \#74.

2. C. Greene, A multiple exchange property for bases, Proc. Amer. Math. Soc. 46 (1974), 155-156.

3. W. Hodge and D. Pedoe, Methods of algebraic geometry. Vol. II, Cambridge Univ. Press, Cambridge, 1952. MR 13, 972.

4. S. L. Kleiman and D. Laksov, Schubert calculus, Amer. Math. Monthly 79 (1972), 1061-1082.

5. D. Lucas, Properties of rank preserving weak maps, Bull. Amer. Math. Soc. 80 (1974), 127-131.

6. L. Mirsky, Transversal theory, Academic Press, New York, 1971. MR 44 \#87.

7. G.C. Rota, On the foundations of combinatorial theory. I. Theory of Mobius functions, Z. Wahrscheinlichkeitstheorie und Verw. Gebiete 2 (1964), 340-368. MR 30 \#4688.

8. Combinatorial theory, old and new, Proc. Internat. Congress Math. (Nice, 1970), vol. 3, Gauthier-Villars, Paris, 1971, pp. 229-233.

9. H. Turnbull, The theory of determinants, matrices, and invariants, Blackie and Son, London, 1928.

10. W. T. Tutte, $A$ homotopy theorem for matroids. I, II, Trans. Amer. Math. Soc. 88 (1958), 144-174. MR 21 \#336.

11. P. Vamos, $A$ necessary and sufficient condition for a matroid to be linear (to appear).

12. B. van der Waerden, Über die Fundamentalen Identitdten der Invariententheorie, Math. Ann. 95 (1926), 706-735. 
13. N. L. White, The bracket ring and combinatorial geometry, Thesis, Harvard University, Cambridge, Mass., 1971.

14. - The bracket ring of a combinatorial geometry. II: Unimodular geometries (to appear).

15. W. Whiteley, Logic and invariant theory. IV: Invariants and syzygies in combinatorial geometry (to appear).

16. A. Young, On quantitative substitutional analysis, Proc. London Math. Soc. (2) 28 (1928), 255-292.

17. O. Zariski and P. Samuel, Commutative algebra. Vol. I, University Ser. in Higher Math., Van Nostrand, Princeton, N.J., 1958. MR 19, 833.

DEPARTMENT OF MATHEMATICS, YALE UNIVERSITY, NEW HAVEN, CONNECTICUT 06520

Current address: Department of Mathematics, University of Florida, Gainesville, Florida 32601 